\title{
Position transitions of polymer-grafted nanoparticles in diblock-copolymer nanocomposites
}

\author{
G. K.Xu, X. Q. Feng* \\ Institute of Biomechanics and Medical Engineering, Department of Engineering Mechanics, Tsinghua University, Beijing \\ 100084, China
}

Received 10 September 2010; accepted in revised form 17 November 2010

\begin{abstract}
Self-assembly of block copolymer/nanoparticle blends has promising applications in the design and fabrication of novel functional nanomaterials. Precise control of the spatial positions of nanoparticles within block copolymer-based nanomaterials is crucial to achieve some special physical properties and functions. Here, we employ the self-consistent field method to theoretically investigate the self-assembly of polymer grafted-nanoparticles in a diblock copolymer. It is found that by varying the size and selectivity of nanoparticles, one can not only produce various self-assembled nanostructures but also modulate the spatial positions of the nanoparticles, either at the copolymer interfaces or in the center of one copolymer phase, within the nanostructures. A denser grafted polymer brush plays a role of shielding effect on nanoparticles and can position them into the center of one copolymer phase. The nanostructural transition we observed is dictated by the competition between entropy and enthalpy. On the basis of a number of simulations, two phase diagrams of self-assembled nanostructures are constructed. This study may be helpful for optimal design of advanced materials with desired nanostructures and enhanced performance.
\end{abstract}

Keywords: nanocomposites, self-assembly, diblock copolymer, polymer-grafted nanoparticle, modeling and simulation

\section{Introduction}

Incorporation of inorganic nanoparticles into a polymeric host has been explored as a highly promising area devoted to improving mechanical, catalytic, magnetic, electrical, and optical properties of nanocomposites [1-8]. The high performance of such nanocomposites makes them attractive for many technologically important applications in, for instance, nanostructured solar cells, photonic band gap materials, optical filters, highly efficient catalysts, and high-density magnetic storage media.

Owing to their rich assortment of ordered morphologies, block copolymers are found to be particularly effective to control the spatial distribution of nanoparticles [9-19], which is crucial to achieve the required properties of nanocomposites. The dispersion of nanoparticles within block copolymer domains is affected by many factors, e.g., the size, shape and selectivity of nanoparticles $[5,9,17,20]$. Among these factors, the chemical interaction between the particles and block copolymers plays the most important role in the self-assembly of nanocomposites. To elaborately adjust this interaction and to prevent macrophase separation, nanoparticles are often grafted by a brush of short polymers which are chemically identical to one component of the block copolymer [10, 12-14, 16]. Recent experiments evidence that various self-assembled nanostructures can be created by incorporating the polymer-grafted nanoparticles into a block copolymer film [10]. Kim et al. [13] reported that in a poly (styrene-b-2-vinylpyridine) (PS-b-P2VP) lamellar morphology, a high grafting PS chain density can drive nanoparticles to move towards the center of

\footnotetext{
${ }^{*}$ Corresponding author, e-mail: fengxq@tsinghua.edu.cn
}

(c) BME-PT 
PS domains, while a low grafting density results in the concentration of nanoparticles at the PS/P2VP interfaces. These findings suggest a facile and versatile means to control self-organization of functionalized nanoparticles and block copolymer blends $[10,12-14,16]$.

Various techniques (e.g., mean-field theory [21], strong segregation theory [22], Monte Carlo [23, 24], dissipative particle dynamics [25, 26], and molecular dynamics [27] methods) have also been developed to investigate the spatial and temporal evolution of complex polymer-nanoparticle systems. Among them, self-consistent field theory (SCFT) has proven to be a powerful method for exploring complex morphologies of block copolymers and blends [28-32]. Small bare nanoparticles can be regarded as large solvent molecules, and their distribution in ordered triblock copolymers has been evaluated by using SCFT [33]. For large nanoparticles in block copolymers, the effect of excluded volume of particles should be taken into account by such methods as a combination of SCFT with density functional theory (DFT) [34-36] and hybrid particle-field method [37]. Very recently, SCFT was further extended to study the self-assembly behavior of polymer-grafted particles in block copolymers. Reister and Fredrickson proposed a novel approach to investigate the phase behavior of a mixture of diblock copolymers and nanoparticles with polymer chains tethered to their surfaces by modeling the grafted particles as star polymers [38]. By regarding particles as an ideal gas, Kim and Matsen explored how to position a single grafted particle in a block copolymer [39].

To date, however, there is still a lack of theoretical study on the spatial distribution of polymer-grafted nanoparticles in a diblock-copolymer lamellar matrix via self-assembly. In particular, it is crucial to quantitatively examine the effect of the grafted polymer brushes and to exploit their role in tuning the nanostructures. In this paper, therefore, we theoretically investigate the self-assembly behavior of polymergrafted nanoparticle/diblock copolymer blends and the spatial position of nanoparticles within lamellar copolymer phases by employing the self-consistent field theory. The grafted nanoparticles are assumed to be sufficiently small so that they can be approximated as solvent molecules in the system [33]. The interactions among the nanoparticles, grafted poly- mers and copolymer matrix are all taken into account in our simulations because of their important roles in the self-assembling progress. Since the performance of a nanocomposite relies strongly on the spatial arrangement of nanoparticles, our attention is focused on how to precisely control the particle position in the nanocomposites. We find that by varying the size and selectivity of nanoparticles as well as the density of the grafted polymer brushes, one can not only obtain different self-assembled nanostructures but also control the spatial positions of the nanoparticles within the nanostructures. Therefore, these factors can be utilized to design nanostructures with a desired particle array, which may endow the material with specific physical properties and functions.

\section{Theoretical model and computational method}

We consider a system containing $\mathrm{AB}$ diblock copolymers and polymer-grafted nanoparticles. Each diblock chain consists of $N_{\mathrm{D}}$ segments, while each grafted chain is composed of $N_{\mathrm{G}}=\beta N_{\mathrm{D}} \mathrm{A}$-type segments. All polymer chains are modeled as flexible Gaussian chains. The volume fraction of A segments in each diblock chain is denoted as $f$. For simplicity, assume that $\mathrm{A}$ and $\mathrm{B}$ segments have the identical volume $\rho_{0}^{-1}$ and statistical length $\alpha$. The particle (P) has a radius $R_{\mathrm{P}}$, and let $\alpha=4 \pi \rho_{0} R_{\mathrm{P}}^{3} / 3 N_{\mathrm{D}}$ denote the volume ratio of a particle to a diblock chain. In this paper, $\alpha$ is taken in the range of $0.01-$ 0.1 to ensure that the nanoparticles are small enough to be approximated as solvent molecules in the system, as assumed by Spontak et al. [33]. For larger particles with a size comparable to the diblock radius of gyration, $R_{\mathrm{g}}=a \sqrt{N_{\mathrm{D}} / 6}$, one should account for the effects of their excluded volume by employing, for instance, the SCFT/DFT approach [34-36]. The number of polymer chains grafted on each particle is denoted as $\sigma$. The volume fractions of diblock copolymers and polymer-grafted particles in the blend are $\varphi_{\mathrm{D}}$ and $\varphi_{\mathrm{GP}}=1-\varphi_{\mathrm{D}}$, respectively. In the SCFT method, the pair interactions between different components are determined by a set of effective chemical potential fields, $W_{\mathrm{I}}(\mathbf{r})$, denoting the intensity of the mean field felt by the species $I$ at position $\mathbf{r}$. The dimensionless free energy of the system is given by Equation (1): 
$F=\frac{N_{\mathrm{D}} F_{0}}{\rho_{0} k_{\mathrm{B}} T V}=-\varphi_{\mathrm{D}} \ln \left(\frac{Q_{\mathrm{D}}}{V \varphi_{\mathrm{D}}}\right)-\frac{\varphi_{\mathrm{GP}}}{(\alpha+\sigma \beta)} \ln \left(\frac{Q_{\mathrm{GP}}}{V \varphi_{\mathrm{GP}}}\right)-\frac{1}{V} \int \mathrm{d} \mathbf{r} \sum_{\mathrm{I}} W_{\mathrm{I}} \phi_{\mathrm{I}}+\frac{1}{2 V} \int \mathrm{d} \mathbf{r} \sum_{\mathrm{I}} \sum_{\mathrm{J} \neq \mathrm{I}} N_{\mathrm{D}} \chi_{\mathrm{IJ}} \phi_{\mathrm{I}} \phi_{\mathrm{J}}$

where $F_{0}$ is the free energy of the system, $V$ the volume of the system, $k_{\mathrm{B}}$ the Boltzmann constant, $T$ the temperature, $\phi_{\mathrm{I}}(\mathbf{r})$ the local volume fraction of species $I$, and $\chi_{\mathrm{IJ}}$ the Flory-Huggins interaction parameter between species $I$ and $J . Q_{\mathrm{D}}$ and $Q_{\mathrm{GP}}$ are the partition functions of a single diblock chain and a polymer-grafted nanoparticle, respectively.

For a diblock chain, the contour variable s increases continuously from 0 at the free end of A-blocks to 1 at the free end of B-blocks. The spatial coordinate $\mathbf{r}$ is normalized by the diblock radius of gyration, $R_{\mathrm{g}}$. The propagator $q_{\mathrm{D}}(\mathbf{r}, s)$ represents the probability of finding the $s N_{\mathrm{D}}$ segment of the diblock chain at position $\mathbf{r}$, which satisfies the modified diffusion equation (see Equtions (2) and (3)):

$$
\begin{aligned}
& \frac{\partial q_{\mathrm{D}}(\mathbf{r}, s)}{\partial s}=\nabla^{2} q_{\mathrm{D}}(\mathbf{r}, s)-W_{\mathrm{I}}(\mathbf{r}) q_{\mathrm{D}}(\mathbf{r}, s) \\
& W_{\mathrm{I}}(\mathbf{r})= \begin{cases}W_{\mathrm{A}}(\mathbf{r}), & 0<s<f \\
W_{\mathrm{B}}(\mathbf{r}), & f<s<1\end{cases}
\end{aligned}
$$

subject to the initial condition $q_{\mathrm{D}}(\mathbf{r}, 0)=1$. The complementary propagator $q_{\mathrm{D}}^{+}(\mathbf{r}, s)$ satisfies an equation similar to Equation (2) except that its right-hand side is multiplied by -1 and the initial condition becomes $q_{\mathrm{D}}^{+}(\mathbf{r}, 1)=1$. The grafted polymer chain propagator $q_{\mathrm{G}}(\mathbf{r}, s)$ is given by Equation (4) [40, 41]:

$$
\frac{\partial q_{\mathrm{G}}(\mathbf{r}, s)}{\partial s}=\beta \nabla^{2} q_{\mathrm{G}}(\mathbf{r}, s)-\beta W_{\mathrm{A}}(\mathbf{r}) q_{\mathrm{G}}(\mathbf{r}, s)
$$

with the initial condition $q_{\mathrm{G}}(\mathbf{r}, 0)=1$ for the free end of the grafted chain at $s=0$. The complementary propagators $q_{\mathrm{G}}{ }^{+}(\mathbf{r}, s)$ obeys an equation similar to Equation (4) except that its right-hand sides is multiplied by -1 with the initial condition being given by Equation (5):

$q_{\mathrm{G}}^{+}(\mathbf{r}, s)=\exp \left[-\alpha W_{\mathrm{P}}(\mathbf{r})\right] q_{\mathrm{G}}(\mathbf{r}, 1)^{\sigma-1}$

In terms of these propagators, the partition functions $Q_{\mathrm{D}}$ and $Q_{\mathrm{GP}}$ are calculated by Equations (6) and (7):

$$
\begin{aligned}
& Q_{\mathrm{D}}=\int \mathrm{d} \mathbf{r} q_{\mathrm{D}}(\mathbf{r}, 1) \\
& Q_{\mathrm{GP}}=\int \mathrm{d} \mathbf{r} \exp \left[-\alpha W_{\mathrm{P}}(\mathbf{r})\right] q_{\mathrm{G}}(\mathbf{r}, 1)^{\sigma}
\end{aligned}
$$

The SCFT method is to find the equilibrium structure of a system by obtaining the minimization of its free energy $F$ as a function of the local densities $\phi_{\mathrm{I}}(\mathbf{r})$ and the mean fields $W_{\mathrm{I}}(\mathbf{r})$ of all components. By minimizing the free energy $F$ with respect to $\phi_{\mathrm{I}}(\mathbf{r})$, one has the following Equations (8)-(10):

$$
\begin{aligned}
& W_{\mathrm{A}}(\mathbf{r})=N_{\mathrm{D}} \chi_{\mathrm{AB}} \phi_{\mathrm{B}}(\mathbf{r})+N_{\mathrm{D}} \chi_{\mathrm{AP}} \phi_{\mathrm{P}}(\mathbf{r})+\xi(\mathbf{r}) \\
& W_{\mathrm{B}}(\mathbf{r})=N_{\mathrm{D}} \chi_{\mathrm{AB}} \phi_{\mathrm{A}}(\mathbf{r})+N_{\mathrm{D}} \chi_{\mathrm{BP}} \phi_{\mathrm{P}}(\mathbf{r})+\xi(\mathbf{r}) \\
& W_{\mathrm{P}}(\mathbf{r})=N_{\mathrm{D}} \chi_{\mathrm{AP}} \phi_{\mathrm{A}}(\mathbf{r})+N_{\mathrm{D}} \chi_{\mathrm{BP}} \phi_{\mathrm{B}}(\mathbf{r})+\xi(\mathbf{r})
\end{aligned}
$$

where $\xi$ is a Lagrange multiplier enhancing the incompressible condition (see Equation (11)):

$$
\phi_{\mathrm{A}}(\mathbf{r})+\phi_{\mathrm{B}}(\mathbf{r})+\phi_{\mathrm{P}}(\mathbf{r})=1
$$

Using the propagators $q_{\mathrm{D}}(\mathbf{r}, s), q_{\mathrm{D}}^{+}(\mathbf{r}, s), q_{\mathrm{G}}(\mathbf{r}, s)$ and $q_{\mathrm{G}}^{+}(\mathbf{r}, s)$, the local densities of different components are calculated respectively by Equtions (12)-(14):

$$
\begin{aligned}
\phi_{\mathrm{A}}(\mathbf{r}) & =\frac{\varphi_{\mathrm{D}} V}{Q_{\mathrm{D}}} \int_{0}^{f} \mathrm{~d} s q_{\mathrm{D}}(\mathbf{r}, s) q_{\mathrm{D}}^{+}(\mathbf{r}, s)+ \\
& +\frac{\varphi_{\mathrm{GP}} \sigma \beta V}{(\alpha+\sigma \beta) Q_{\mathrm{GP}}} \int_{0}^{1} \mathrm{~d} s q_{\mathrm{G}}(\mathbf{r}, s) q_{\mathrm{G}}^{+}(\mathbf{r}, s) \\
\phi_{\mathrm{B}}(\mathbf{r}) & =\frac{\varphi_{\mathrm{D}} V}{Q_{\mathrm{D}}} \int_{f}^{1} \mathrm{~d} s q_{\mathrm{D}}(\mathbf{r}, s) q_{\mathrm{D}}^{+}(\mathbf{r}, s) \\
\phi_{\mathrm{P}}(\mathbf{r}) & =\frac{\varphi_{\mathrm{GP}} \alpha V}{(\alpha+\sigma \beta) Q_{\mathrm{GP}}} \exp \left[-\alpha W_{\mathrm{P}}(\mathbf{r})\right] q_{\mathrm{G}}(\mathbf{r}, 1)^{\sigma}
\end{aligned}
$$

Equations (8)-(14) form a closed set of equations that can be solved self-consistently in real space. To obtain the equilibrium nanostructure, we solve these equations by using the combinatorial screening technique of Drolet and Fredrickson [30, 42] implemented with a highly stable and accurate numerical algorithm [43, 44]. All the simulations are performed in a two-dimensional square lattice with periodic boundary conditions. Since the equilibrium morphology is somewhat influenced by the simulation box size [45], two methods have been often adopted to minimize the free energy with respect to the box size. One is to fix the simulation cell shape and to gradually adjust the cell size [46, 
47], while in the other, both the shape and size of the cell are adjusted [48]. In our simulations, we use the former method to achieve an equilibrium structure by changing the square cell size, $a$, such that $\partial F\left(a_{\min }\right) / \partial a=0$. The system is thought to have reached equilibrium when the relative difference between the free energies of the system at two neighboring iteration steps has been smaller than $0.0001 \%$. More details on the numerical implementation of SCFT can be found in the literature [30, 42-44].

\section{Results and discussion}

In this study, the incompatibility between A- and Bblocks is set as $N_{\mathrm{D}} \chi_{\mathrm{AB}}$. The interaction parameters of A- and B-blocks with the particle are respectively characterized by $N_{\mathrm{D}} \chi_{\mathrm{AP}}=\varepsilon N_{\mathrm{D}} \chi_{\mathrm{AB}}$ and $N_{\mathrm{D} \chi_{\mathrm{BP}}}=$ $(1-\varepsilon) N_{\mathrm{D}} \chi_{\mathrm{AB}}$, in which $\varepsilon$ denotes the particle selectivity [33]. If $\varepsilon>0.5$, the particle has a preferable interaction to B-blocks (i.e., B-selective). $\varepsilon<0.5$ indicates an attraction interaction between the particle and A-blocks (i.e., A-selective). For a neutral particle, one has $\varepsilon=0.5$. Unless specified otherwise, the following representative values are used: $f=0.5, \sigma=3, \Delta s=0.01, \alpha=0.08$ and $\varepsilon=0.5$. We have carried out a large number of simulations by changing the number $\sigma$ of polymer chains tethered on each particle in the range from 2 to 6 . The results demonstrate that $\sigma=3$ can well describe the effect of grafted polymers and shorten the calculation time. The formation of various patterns depends on the volume fraction of polymer-grafted nanoparti-
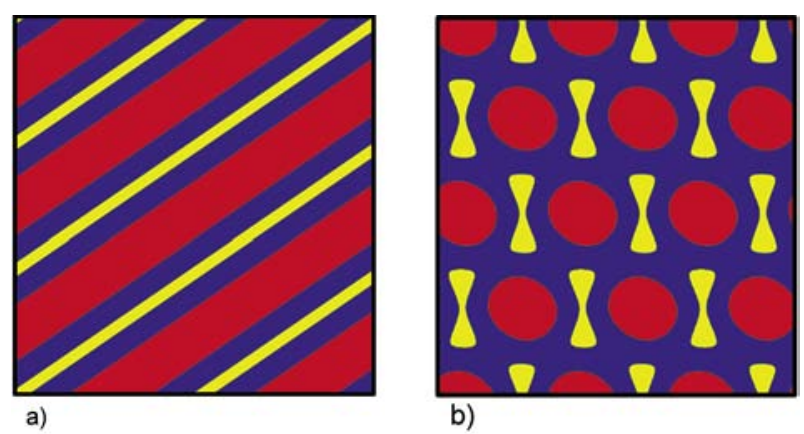

Figure 1. Aggregation morphologies of polymer-grafted nanoparticle and diblock copolymer blends under different concentrations of polymer-grafted nanoparticles: (a) lamellar pattern under $\varphi_{\mathrm{GP}}=0.15$ and (b) hexagonally packed pattern under $\varphi_{\mathrm{GP}}=$ 0.4 . The concentration of grafted polymers is taken as $\varphi_{\mathrm{G}}=2 \varphi_{\mathrm{GP}} / 3$ in these two cases. The blue, red, and yellow colors represent A-blocks, Bblocks, and particles, respectively. cles [10]. In our previous work [49], it has been demonstrated that a transition from the lamellar $\left(\varphi_{\mathrm{GP}}=0.15\right.$, Figure 1a) to hexagonally packed $\left(\varphi_{\mathrm{GP}}=\right.$ 0.4 , Figure $1 \mathrm{~b}$ ) pattern occurs as the grafted particle concentration $\varphi_{\mathrm{GP}}$ increases. In this paper, our attention is focused on how to control the spatial distribution of nanoparticles in a diblock-copolymer lamellar morphology, because of its wide applications in nanocomposites [2, 5, 9, 12-14]. In what follows, we will take $\varphi_{\mathrm{GP}}=0.15$.

\subsection{Effect of nanoparticle size}

We first examine the size effect of nanoparticles on the equilibrium structure. The concentration of grafted A-type polymers is fixed at $\varphi_{\mathrm{G}}=0.075$ in this subsection. The tethered A-type polymers will prevent the presence of the nanoparticles in $\mathrm{B}$ domains because of the repulsive interaction between the grafted A chains and the B segments. The self-assembled nanostructures of the blends are shown in Figure 2a, 2c, 2e under three representative sizes of nanoparticles. It is seen from Figure $2 \mathrm{a}$ that when the particles (yellow) are very small (e.g., $\alpha=0.01$ ), they will have a concentration at the copolymer interfaces. Particles with mediate sizes (e.g., $\alpha=0.02$ ) can be observed both at the copolymer interfaces and in the center of A domains (Figure 2c). Bigger particles will mainly reside in the center of A domains, as illustrated in Figure 2e, where we take $\alpha=0.08$. To reveal the structures more clearly, the density profiles of different components (nanoparticles, A- and B-blocks) with two periods are plotted along the direction perpendicular to the interfaces in the lamellar structure (Figure $2 b, 2 d, 2 f)$. A period refers to the region between the centers of two neighboring B domains. In Figure $2 b$, the particle density profile in each period has two peaks, located at the $\mathrm{A} / \mathrm{B}$ interfaces, indicating that the nanoparticles are mainly distributed in A domains with a concentration at the copolymer interfaces. In Figure 2d, the particle density profile has three peaks in each period, which are located at the copolymer interfaces and in the center of $\mathrm{A}$ domains, respectively, but the spatial distribution within A domains is relatively even. In the case of the largest particles we study, the particle density profile has only one peak in the center of each period (Figure 2f), representing the aggregation of particles in the center of A domains. 


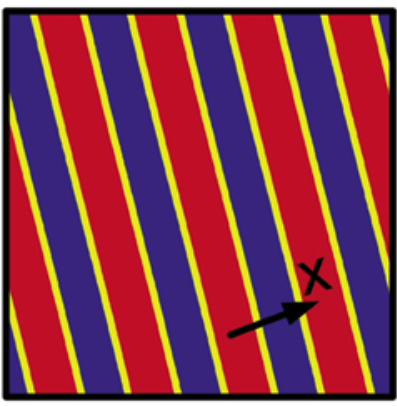

a)

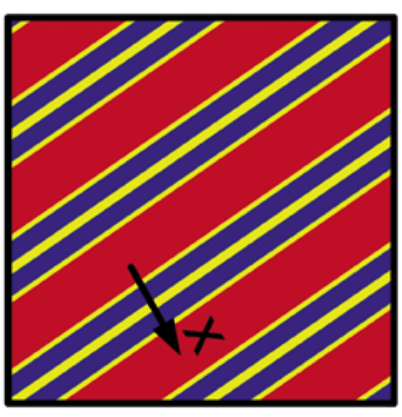

c)

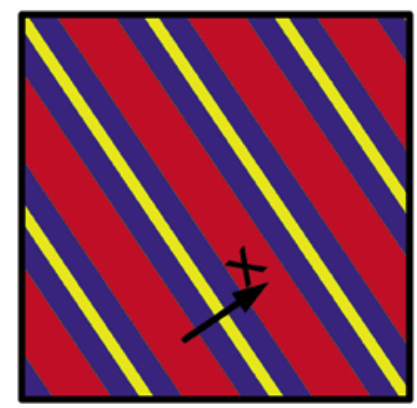

e)
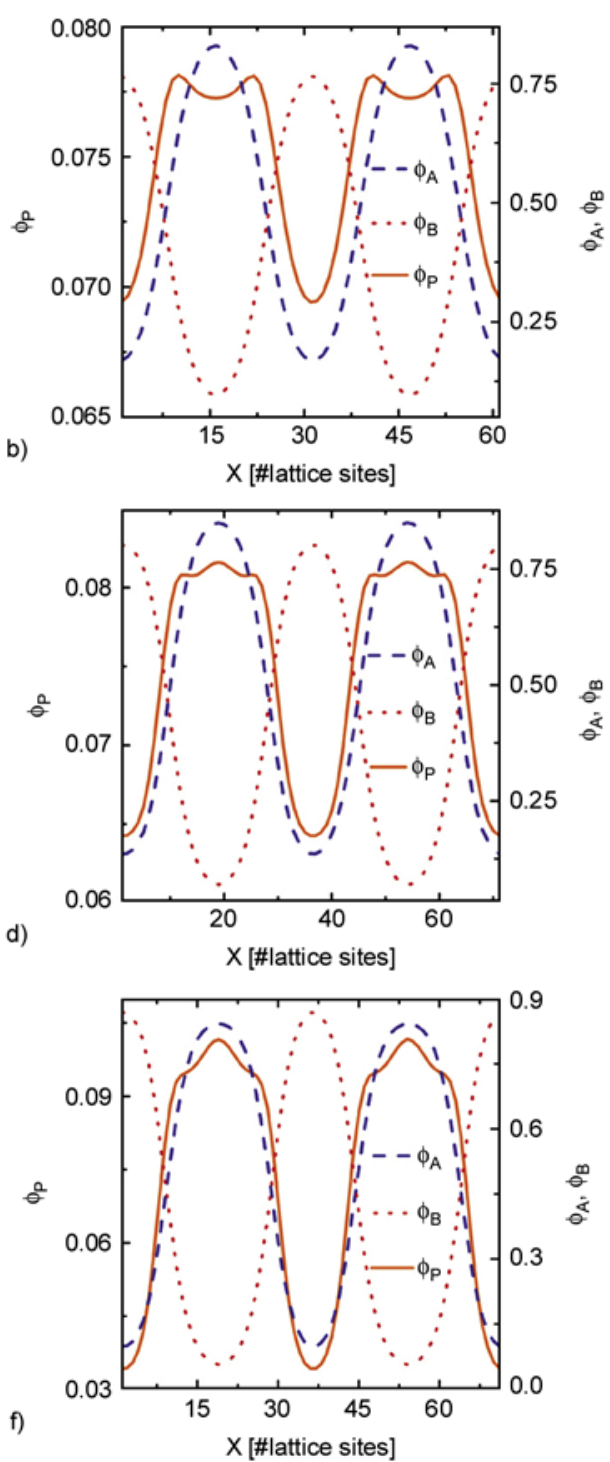

Figure 2. Influence of the particle size $\alpha$ on the self-assembled nanostructures of polymer-grafted nanoparticle and diblock copolymer blends: (a) $\alpha=0.01$, (c) $\alpha=0.02$ and (e) $\alpha=0.08$. The blue, red and yellow colors represent A-blocks, B-blocks, and nanoparticles, respectively. The corresponding two period-density profiles of A-blocks $\left(\phi_{\mathrm{A}}\right)$, B-blocks $\left(\phi_{\mathrm{B}}\right)$ and particles $\left(\phi_{\mathrm{P}}\right)$ are plotted in (b), (d) and (f) as the arrows pointed in the (a), (c) and (e), respectively.

The size-induced particle position transitions are more clearly schematized in Figure 3. This transition can be understood by considering the competition between the translational entropy of nanoparticles and the conformational entropy of copolymers. For large particles, the copolymers push them towards the center of A domains to gain more conformational entropy, which overcomes the loss of particle translational entropy. Under a fixed volume fraction, the number of particles increases as their size reduces. Therefore, small particles are mainly dispersed in the preferred domains and their translational entropy dominates the final nanostructure. This morphological transition with the variation of the particle size is consistent with recent experimental observations [9]. In addition, Balazs et al. [34] drawn a similar conclusion in their study of the structures of bare particle/diblock copolymer blends.

\subsection{Effect of grafted polymer brush}

In this subsection, we quantitatively investigate the effect of grafted polymer brushes, which serve as a shield on nanoparticles and play a significant role in the self-assembling process of the blends. The concentration of grafted polymers, $\varphi_{\mathrm{G}}$, is a function of the grafted chain length $\beta$ and the number $\sigma$ of grafted polymers on each particle. Under a fixed 


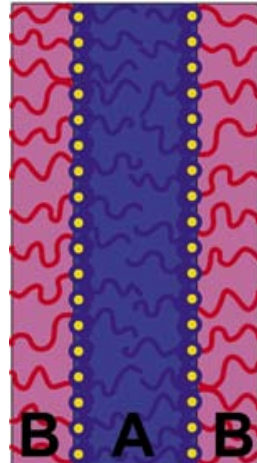

a)
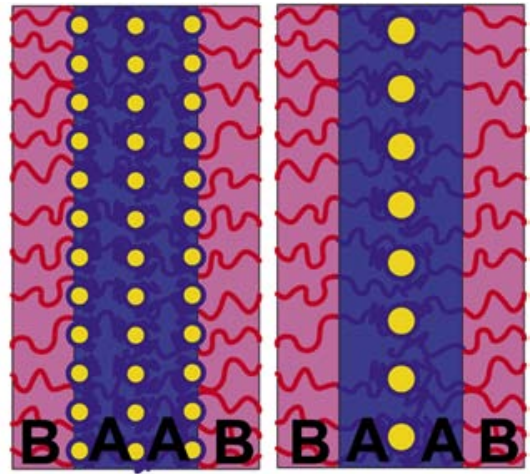

b)

Figure 3. Schematic diagrams showing the major positions of nanoparticles under different conditions. Small nanoparticles segregate to the copolymer interfaces (a), while large nanoparticles locate in the center of A domains (c). A combination of the two particle positions occurs at the mediate particle size (b). The blue, red and yellow colors represent A-blocks, B-blocks, and particles, respectively.

value of $\sigma=3$, therefore, $\varphi_{\mathrm{G}}$ depends mainly on the attached chain length $\beta$. A larger value of $\varphi_{\mathrm{G}}$ stands for a denser grafted polymer brush and a stronger shielding effect. For several representative values of $\varphi_{\mathrm{G}}$, the density profiles of particles are compared in Figure 4, where the notations $\mathrm{CA}$ and $\mathrm{CB}$ respectively represent the centers of $\mathrm{A}$ and $\mathrm{B}$ domains. It is seen that in the case of a sparse polymer brush (e.g., $\varphi_{\mathrm{G}}=0.03$ ), most nanoparticles mainly reside in the A domains but, simultaneously, there is an enrichment at the copolymer interfaces, which weakens the enthalpic interaction between A- and B-blocks. When the polymer brushes are denser (e.g., $\varphi_{\mathrm{G}}=0.09$ ), particles are mainly embedded in the center of A domains. A combination of the above two distributions of nanoparticles occurs at the mediate range of the grafted polymer concentration (e.g., $\varphi_{\mathrm{G}}=0.06$ ). At a small value of $\varphi_{\mathrm{G}}$, the particles move towards the copolymer interfaces, effectively reducing the interfacial interaction between A- and B-blocks at A/B interfaces. However, under a larger concentration of grafted polymers, the particles are almost completely shielded by the denser polymer brushes. As a result, the nanoparticles migrate into the center of A domains, inducing a decrease of enthalpic interaction between the grafted A brushes and B-blocks and also an increase of the entropy of copolymers. Recently, Chiu et al. [16] experimentally observed that A-grafted nanoparticles locate only in the center of A domains, irre-

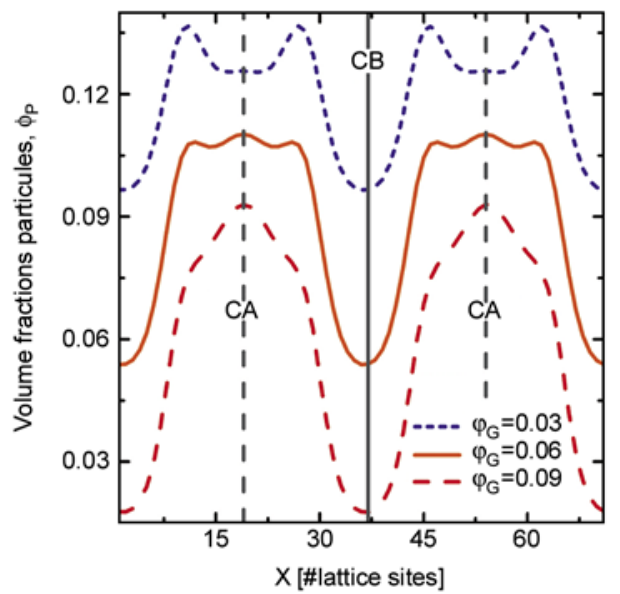

Figure 4. Two period-density profiles of particles are plotted with various grafted polymer concentrations: $\varphi_{\mathrm{G}}=0.03,0.06$ and 0.09 . The gray dashed line represents the center of A domains (CA), and the gray solid line shows the center of $\mathrm{B}$ domains (CB).

spective of the particle size. This finding can be interpreted by the shielding effect of the denser grafted polymer brushes used in their experiments, as observed in our simulations. Therefore, it is conclusive that by appropriate design of the grafted polymer brush and the particle size, nanoparticles can be precisely positioned in a nanostructure, either in the required domains or interfaces, depending on the need for specific physical properties and functions.

\subsection{Effect of selectivity}

Enthalpy often dominates the self-assembly and microphase segregation of a system, especially at the nanoscale. Therefore, we here investigate the effect of enthalpic interactions between nanoparticles and different components of copolymers on the equilibrium structure. The particle selectivity $\varepsilon$ is varied to study this effect under a fixed polymer brush concentration, $\varphi_{\mathrm{G}}=0.075$. Figure 5 shows the density profiles of nanoparticles under several representative values of $\varepsilon$. It is seen from Figure $2 \mathrm{f}$ that neutral particles $(\varepsilon=0.5)$ segregate to the center of A domains. When the particles are highly B-selective (e.g., $\varepsilon=0.875$ ), they tend to reside in B domains because of their preferable interaction, whereas the incompatibility between grafted A brushes and Bblocks excludes the presence on nanoparticles in $\mathrm{B}$ domains. The competition of these two factors induces the enrichment of particles at $\mathrm{A} / \mathrm{B}$ inter- 


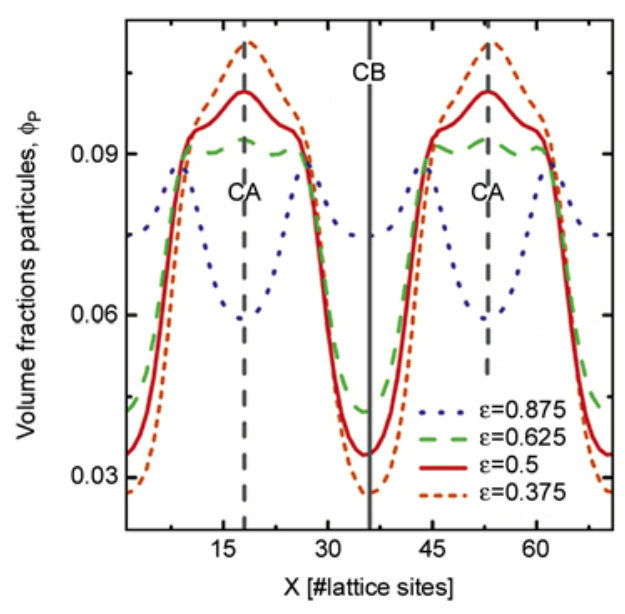

Figure 5. Two period-density profiles of particles under various particle selectivities: $\varepsilon=0.875,0.625$, 0.5 , and 0.375. $\varepsilon>0.5$ and $\varepsilon<0.5$ indicate that the particles are B-selective and A-selective, respectively, while $\varepsilon=0.5$ indicates neutral particles. The gray dashed line represents the center of A domains (CA), and the gray solid line shows the center of $\mathrm{B}$ domains $(\mathrm{CB})$.

faces. In the case of $\varepsilon=0.625$, there are three peaks in each period of the particle density profile, indicating that particles reside both in the center of $\mathrm{A}$ domains and at the A/B interfaces. For A-selective particles (e.g., $\varepsilon=0.375$ ), they mainly locate in the center of A domains owing to the favorable interaction between the particles and A-blocks in spite of the loss in the particle translational entropy.

To quantitatively explore the physical mechanisms underlying the formation of the above-described nanostructures, we calculate the contributions of entropy and enthalpy to the total free energy. In Equation (1), the first three terms of the right-hand side represent the energy associated with entropy while the last term corresponds to enthalpy. The dimensionless total free energy of the system $(F)$, the contribution of entropy $\left(F_{\mathrm{S}}\right)$ and enthalpy $\left(F_{\mathrm{E}}\right)$ are compared in Table 1 for under several representative values of the selectivity coefficient $\varepsilon$. As $\varepsilon$ decreases, the entropic contribution to the total free energy increases because the concentration of nanoparticles at the centre of A domains leads to a partial loss of their translational entropy. However, the enthalpic interaction decreases since the total contact area between copolymers and particles reduces as the particles migrate from the $\mathrm{A} / \mathrm{B}$ interfaces to the center of A domains. For instance, the difference of the entropic contribution to the free energy between two situations of $\varepsilon=0.875$ and $\varepsilon=0.375$ is
Table 1. Comparison of the dimensionless free energy $F$ of the system at equilibrium under several representative values of the selectivity coefficient, $\varepsilon . F_{\mathrm{S}}$ and $F_{\mathrm{E}}$ stand for the entropic and enthalpic contributions to the free energy, respectively.

\begin{tabular}{|c|c|c|c|}
\hline $\begin{array}{c}\text { Selectivity } \\
\boldsymbol{\varepsilon}\end{array}$ & $\begin{array}{c}\text { Entropic } \\
\text { contribution } \\
\mathbf{F}_{\mathbf{S}}\end{array}$ & $\begin{array}{c}\text { Enthalpic } \\
\text { contribution } \\
\mathbf{F}_{\mathbf{E}}\end{array}$ & $\begin{array}{c}\text { Total free } \\
\text { energy } \\
\mathbf{F}\end{array}$ \\
\hline 0.875 & 1.151 & 2.791 & 3.942 \\
\hline 0.625 & 1.241 & 2.654 & 3.895 \\
\hline 0.500 & 1.309 & 2.547 & 3.856 \\
\hline 0.375 & 1.399 & 2.414 & 3.813 \\
\hline
\end{tabular}

0.248 , whereas the corresponding enthalpic contribution to the free energy has a difference of -0.377 . In comparison with the entropic contribution, the enthalpy dominates the particle distribution within the self-assembled nanostructures.

\subsection{Phase diagrams of self-assembled nanostructures}

To further reveal the dependence of the particle location upon the particle size, particle selectivity and grafted polymer concentration, two phase diagrams of the self-assembled lamellar structures are provided in Figure 6 based on a number of simulations. The gray lines represent the transition boundaries, and the insets delineate particle positions. Corresponding to distinctly different particle positions within the lamellar morphology, each diagram is divided into three regions, marked by $\mathrm{I},(\mathrm{I}+\mathrm{CA})$ and CA, respectively. The notation I stands for the preferential segregation of nanoparticles to $\mathrm{A} / \mathrm{B}$ interfaces, while the notation CA stands for the concentration of particles in the center of A domains. It is clearly seen from Figure 6a that the particle position transitions in the order of $\mathrm{I} \rightarrow(\mathrm{I}+\mathrm{CA}) \rightarrow \mathrm{CA}$ can be achieved by increasing either the particle size or the concentration of grafted polymer brushes. With increasing $\varepsilon$, the morphological transitions take place in the order of $\mathrm{CA} \rightarrow(\mathrm{I}+\mathrm{CA}) \rightarrow \mathrm{I}$, as shown in Figure 6b, as a result of the increased preferential interaction between the nanoparticles and B segments. Such phase diagrams provide a facile tool to predict the particle distribution in a block-copolymer lamellar morphology under specified conditions.

\section{Conclusions}

In summary, we report a theoretical investigation of the spatial distribution of polymer-tethered nano- 

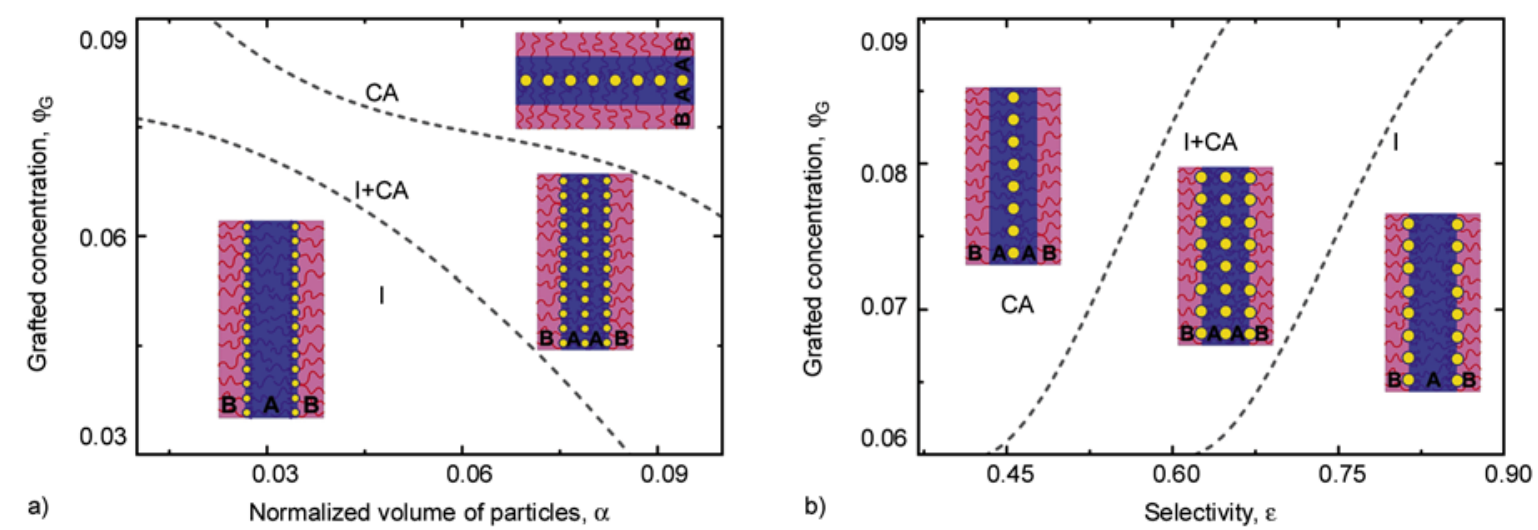

Figure 6. Phase diagrams illustrating the dependence of particle position on the particle size $\alpha$, particle selectivity $\varepsilon$, and grafted polymer concentration $\varphi_{\mathrm{G}}$. (a) Particle size-grafted concentration phase diagram. (b) Particle selectivitygrafted concentration phase diagram. The insets show the preferable positions of particles. The notations I and CA stand for particles mainly locating at the copolymer interfaces and in the center of A domains, respectively.

particles within the lamellar diblock-copolymer matrix. Our study suggests that by appropriate design of the grafted polymer brush, the nanoparticle size and surface treatment (i.e., selectivity), nanoparticles can be precisely positioned in nanocomposites with a desired array for specific properties and applications. Through systematic SCFT simulations, we constructed two phase diagrams describing the dependence of the spatial distribution of nanoparticles on their size and selectivity and the concentration of the grafted polymers. The observed position transition of particles is dictated by the competition between entropy and enthalpy in the system. The present method can also account for other factors (e.g., the composition of copolymers [50], shape [20] and concentration [10, 51] of nanoparticles) that can be utilized to fabricate more delicate nanostructures. This study is helpful for optimal design of self-assembled composites with desired nanostructures and enhanced properties.

\section{Acknowledgements}

The supports from the National Natural Science Foundation of China (Grants Nos. 10972121, 10732050 and 10772093), the Ministry of Education (SRFDP 20090002110047), and the 973 program of MOST (2010CB631005) are acknowledged.

\section{References}

[1] Balazs A. C., Emrick T., Russell T. P.: Nanoparticle polymer composites: Where two small worlds meet. Science, 314, 1107-1110 (2006).

DOI: $\underline{10.1126 / \text { science. } 1130557}$
[2] Bockstaller M. R., Thomas E. L.: Proximity effects in self-organized binary particle-block copolymer blends. Physical Review Letters, 47, 166106/1-166106/4 (2004).

DOI: $10.1103 /$ PhysRevLett.93.166106

[3] Liff S. M., Kumar N., McKinley G. H.: High-performance elastomeric nanocomposites via solventexchange processing. Nature Materials, 6, 76-83 (2007).

DOI: $10.1038 /$ nmat1798

[4] Warren S. C., Messina L. C., Slaughter L. S., Kamperman M., Zhou Q., Gruner S. M., DiSalvo F. J., Wiesner U.: Ordered mesoporous materials from metal nanoparticle-block copolymer self-assembly. Science, 320, 1748-1752 (2008).

DOI: $10.1126 /$ science. 1159950

[5] Bockstaller M. R., Mickiewicz R. A., Thomas E. L.: Block copolymer nanocomposites: Perspectives for tailored functional materials. Advanced Materials, 17, 1331-1349 (2005).

DOI: $10.1002 / \mathrm{adma} .200500167$

[6] Thompson R. B., Rasmussen K. Ø., Lookman T.: Origins of elastic properties in ordered block copolymer/ nanoparticle composites. Nano Letters, 6, 2455-2459 (2006).

DOI: $10.1021 / \mathrm{n} 1048407 \mathrm{f}$

[7] Meli L., Green P. F.: Aggregation and coarsening of ligand-stabilized gold nanoparticles in poly(methl methacrylate) thin films. ACS Nano, 2, 1305-1312 (2008).

DOI: $10.1021 / \mathrm{nn} 800045 \mathrm{~s}$

[8] Akcora P., Liu H., Kumar S. K., Moll J., Li Y., Benicewicz B. C., Schadler L. S., Acehan D., Panagiotopoulos A. Z., Pryamitsyn V., Ganesan V., Ilavsky J., Thiyagarajan P., Colby R. H., Douglas J. F.: Anisotropic self-assembly of spherical polymergrafted nanoparticles. Nature Materials, 8, 354-359 (2009).

DOI: $10.1038 /$ NMAT2404 
[9] Bockstaller M. R., Lapetnikov Y., Margel S., Thomas E. L.: Size-selective organization of enthalpic compatibilized nanocrystals in ternary block copolymer/particle mixture. Journal of the American Chemical Society, 125, 5276-5277 (2003).

DOI: $10.1021 / \mathrm{ja} 034523 \mathrm{t}$

[10] Kim B. J., Chiu J. J., Yi G-R., Pine D. J., Kramer E. J.: Nanoparticle-induced phase transitions in diblockcopolymer films. Advanced Materials, 17, 2618-2622 (2005).

DOI: $10.1002 / \mathrm{adma} .200500502$

[11] Lin Y., Böker A., He J., Sill K., Xiang H., Abetz C., Li X., Wang J., Emrick T., Long S., Wang Q., Balazs A., Russell T. P.: Self-directed self-assembly of nanoparticle/copolymer mixtures. Nature, 434, 55-59 (2005). DOI: $10.1038 /$ nature 03310

[12] Chiu J. J., Kim B. J., Kramer E. J., Pine D. J.: Control of nanoparticle location in block copolymers. Journal of the American Chemical Society, 127, 5036-5037 (2005).

DOI: $10.1021 / \mathrm{ja} 050376 \mathrm{i}$

[13] Kim B. J., Bang J., Hawker C. J., Kramer E. J.: Effect of areal chain density on the location of polymer-modified gold nanoparticles in a block copolymer template. Macromolecules, 39, 4108-4114 (2006).

DOI: $10.1021 / \mathrm{ma} 060308 \mathrm{w}$

[14] Kim B. J., Fredrickson G. H., Kramer E. J.: Effect of polymer ligand molecular weight on polymer-coated nanoparticle location in block copolymers. Macromolecules, 41, 436-447 (2008).

DOI: $10.1021 / \mathrm{ma} 701931 \mathrm{z}$

[15] Kang H., Detcheverry F. A., Mangham A. N., Stoykovich M. P., Daoulas K. C., Hamers R. J., Müller M., de Pablo J. J., Nealey P. F.: Hierarchical assembly of nanoparticle superstructures from block copolymernanoparticle composites. Physical Review Letters, 100, 148303/1-148303/4 (2008).

DOI: $10.1103 /$ PhysRevLett.100.148303

[16] Chiu J. J., Kim B. J., Yi G-R., Bang J., Kramer E. J., Pine D. J.: Distribution of nanoparticles in lamellar domains of block copolymers. Macromolecules, 40, 3361-3365 (2007).

DOI: $10.1021 / \mathrm{ma} 061503 \mathrm{~d}$

[17] Warren S. C., DiSalvo F. J., Wiesner U.: Nanoparticletuned assembly and disassembly of mesostructured silica hybrids. Nature Materials, 6, 156-161 (2007). DOI: $10.1038 /$ nmat 1819

[18] Hickey R. J., Sanchez-Gaytan B. L., Cui W., Composto R. J., Fryd M., Wayland B. B., Park S-J.: Morphological transitions of block-copolymer bilayers via nanoparticle clustering. Small, 6, 48-51 (2010).

DOI: $10.1002 / \mathrm{smll} .200901266$

[19] Darling S. B.: Directing the self-assembly of block copolymers. Progress in Polymer Science, 32, 11521204 (2007).

DOI: 10.1016/j.progpolymsci.2007.05.004
[20] Kim J. U., O’Shaughnessy B.: Morphology selection of nanoparticle dispersions by polymer media. Physical Review Letters, 89, 238301/1-238301/4 (2002). DOI: $10.1103 /$ PhysRevLett.89.238301

[21] Pryamtisyn V., Ganesan V., Panagiotopoulos A. Z., Liu H., Kumar S. K.: Modeling the anisotropic self-assembly of spherical polymer-grafted nanoparticles. Journal of Chemical Physics, 131, 221102/1-221102/4 (2009).

DOI: $10.1063 / 1.3267729$

[22] Pryamitsyn V., Ganesan V.: Strong segregation theory of block copolymer-nanoparticle composites. Macromolecules, 39, 8499-8510 (2006).

DOI: $10.1021 / \mathrm{ma} 0613382$

[23] Wang Q., Nealey P. F., de Pablo J. J.: Behavior of single nanoparticle/homopolymer chain in ordered structures of diblock copolymers. Journal of Chemical Physics, 118, 11278-11285 (2003).

DOI: $10.1063 / 1.1575207$

[24] Huh J., Ginzburg V. V., Balazs A. C.: Thermodynamic behavior of particle/diblock copolymer mixtures: Simulation and theory. Macromolecules, 33, 8085-8096 (2000). DOI: $10.1021 / \mathrm{ma} 000708 \mathrm{y}$

[25] Huang J., Wang Y.: Control of aggregation of nanoparticles by double-hydrophilic block copolymers: A dissipative particle dynamics study. The Journal of Physical Chemistry B, 111, 7735-7741 (2007).

DOI: $10.1021 / j p 070160 \mathrm{y}$

[26] Huang J., Luo M., Wang Y.: Dissipative particle dynamics simulation on a ternary system with nanoparticles, double-hydrophilic block copolymers, and solvent. The Journal of Physical Chemistry B, 112, 6735-6741 (2008).

DOI: $10.1021 / j p 710567 \mathrm{f}$

[27] Schultz A. J., Hall C. K., Genzer J.: Computer simulation of block copolymer/nanoparticle composites. Macromolecules, 38, 3007-3016 (2005).

DOI: $10.1021 / \mathrm{ma} 0496910$

[28] Matsen M. W.: The standard Gaussian model for block copolymer melts. Journal of Physics: Condensed Matter, 14, R21-R47 (2002). DOI: $10.1088 / 0953-8984 / 14 / 2 / 201$

[29] Matsen M. W.: Stabilizing new morphologies by blending homopolymer with block copolymer. Physical Review Letters, 74, 4225-4228 (1995). DOI: $10.1103 /$ PhysRevLett.74.4225

[30] Drolet F., Fredrickson G. H.: Combinatorial screening of complex block copolymer assembly with self-consistent field theory. Physical Review Letters, 83, 4317-4320 (1999). DOI: $10.1103 /$ PhysRevLett.83.4317

[31] Xu G. K., Li Y., Li B., Feng X. Q., Gao H.: Self-assembled lipid nanostructures encapsulating nanoparticles in aqueous solution. Soft Matter, 5, 3977-3983 (2009). DOI: $10.1039 / \mathrm{b} 906918 \mathrm{f}$ 
[32] Xu G. K., Feng X. Q., Li Y.: Self-assembled nanostructures of homopolymer and diblock copolymer blends in a selective solvent. The Journal of Physical Chemistry B, 114, 1257-1263 (2010).

DOI: $10.1021 / j p 908823 \mathrm{~h}$

[33] Spontak R. J., Shankar R., Bowman M. K., Krishnan A. S., Hamersky M. W., Samseth J., Bockstaller M. R., Rasmussen K. Ø.: Selectivity- and size-induced segregation of molecular and nanoscale species in microphase-ordered triblock copolymers. Nano Letters, 6, 2115-2120 (2006).

DOI: $10.1021 / \mathrm{nl} 061205 \mathrm{u}$

[34] Thompson R. B., Ginzburg V. V., Matsen M. W., Balazs A. C.: Predicting the mesophases of copolymernanoparticle composites. Science, 292, 2469-2472 (2001).

DOI: $\underline{10.1126 / \text { science. } 1060585}$

[35] Thompson R. B., Ginzburg V. V., Matsen M. W., Balazs A. C.: Block copolymer-directed assembly of nanoparticles: Forming mesoscopically ordered hybrid materials. Macromolecules, 35, 1060-1071 (2002). DOI: $10.1021 / \mathrm{ma} 011563 \mathrm{~d}$

[36] Lee J. Y., Shou Z., Balazs A. C.: Modeling the selfassembly of copolymer-nanoparticle mixtures confined between solid surfaces. Physical Review Letters, 91, 136103/1-136103/4 (2003).

DOI: 10.1103/PhysRevLett.91.136103

[37] Sides S. W., Kim B. J., Kramer E. J., Fredrickson G. H.: Hybrid particle-field simulations of polymer nanocomposites. Physical Review Letters, 96, 250601/1-250601/4 (2006).

DOI: 10.1103/PhysRevLett.96.250601

[38] Reister E., Fredrickson G. H.: Phase behavior of a blend of polymer-tethered nanoparticles with diblock copolymers. Journal of Chemical Physics, 123, 214903/1-214903/13 (2005).

DOI: $10.1063 / 1.2117008$

[39] Kim J. U., Matsen M. W.: Positioning Janus nanoparticles in block copolymer scaffolds. Physical Review Letters, 102, 078303/1-078303/4 (2009). DOI: 10.1103/PhysRevLett.102.078303

[40] Patel D. M., Fredrickson G. H.: Quenched and annealed disorder in randomly grafted copolymer melts. Physical Review E, 68, 051802/1-051802/4 (2003). DOI: 10.1103/PhysRevE.68.051802

[41] Zhang L., Lin J., Lin S.: Aggregate morphologies of amphiphilic graft copolymers in dilute solution studied by self-consistent field theory. The Journal of Physical Chemistry B, 111, 9209-9217 (2007).

DOI: 10.1021/jp0684291
[42] Drolet F., Fredrickson G. H.: Optimizing chain bridging in complex block copolymers. Macromolecules, 34, 5317-5324 (2001). DOI: $10.1021 / \mathrm{ma} 0100753$

[43] Tzeremes G., Rasmussen K. Ø., Lookman T., Saxena A.: Efficient computation of the structural phase behavior of block copolymers. Physical Review E, 65, 041806/1-041806/5 (2002).

DOI: 10.1103/PhysRevE.65.041806

[44] Sides S. W., Fredrickson G. H.: Parallel algorithm for numerical self-consistent field theory simulations of block copolymer structure. Polymer, 44, 5859-5866 (2003).

DOI: $10.1016 / \mathrm{S} 0032-3861(03) 00606-2$

[45] Bohbot-Raviv Y., Wang Z-G.: Discovering new ordered phases of block copolymers. Physical Review Letters, 85, 3428-3431 (2000). DOI: $10.1103 /$ PhysRevLett.85.3428

[46] Jiang Y., Yan X., Liang H., Shi A-C.: Effect of polydispersity on the phase diagrams of linear ABC triblock copolymers in two dimensions. The Journal of Physical Chemistry B, 109, 21047-21055 (2005).

DOI: 10.1021/jp052902y

[47] Kou D., Jiang Y., Liang H.: Microstructures from a mixture of $\mathrm{ABC}$ 3-miktoarm star terpolymers and homopolymers in two-dimensional space. The Journal of Physical Chemistry B, 110, 23557-23563 (2006). DOI: $10.1021 / \mathrm{jp} 063695 \mathrm{k}$

[48] Tyler C. A., Morse D. C.: Stress in self-consistent-field theory. Macromolecules, 36, 8184-8188 (2003). DOI: $10.1021 / \mathrm{ma} 034601 \mathrm{x}$

[49] Xu G. K., Feng X. Q., Yu S. W.: Controllable nanostructural transitions in grafted nanoparticle-block copolymer composites. Nano Research, 3, 356-362 (2010). DOI: $10.1007 / \mathrm{s} 12274-010-1039-8$

[50] Lo C-T., Lee B., Pol V. G., Dietz Rago N. L., Seifert S., Winans R. E., Thiyagarajan P.: Effect of molecular properties of block copolymers and nanoparticles on the morphology of self-assembled bulk nanocomposites. Macromolecules, 40, 8302-8310 (2007).

DOI: $10.1021 / \mathrm{ma} 070835 \mathrm{v}$

[51] Kim B. J., Fredrickson G. H., Bang J., Hawker C. J., Kramer E. J.: Tailoring core-shell polymer-coated nanoparticles as block copolymer surfactants. Macromolecules, 42, 6193-6201 (2009).

DOI: $10.1021 / \mathrm{ma9009406}$ 